\title{
A Novel Linear Programming Optimization Coverage Scheme Based on Probability model
}

\author{
Ya-ning Yan ${ }^{1, a, *}$ and Jing $\mathrm{Li}^{1, \mathrm{~b}}$ \\ ${ }^{1}$ ZTE Telecommunications College, Xi’an Peihua University, Xi’an 710065, China \\ a394811297@qq.com, b285149439@qq.com \\ *corresponding author
}

Keywords: Wireless sensor network; Coverage rate; Redundancy; Probabilistic model; Network lifetime

\begin{abstract}
In this paper, we present an optimized coverage algorithm based on dynamic parameter model.Through the definition of dynamic parameter coverage model, the expected value and tolerance of the coverage of the sensor nodes and the expected value formula for the first coverage of the target node are given. In the aspect of network energy, the communication path is optimized by the node state scheduling strategy, so that the energy matching of sensor nodes is effectively realized, and the energy consumption of sensor nodes is suppressed. Simulation results show that the proposed algorithm can not only improve the coverage and QoS of the network, but also effectively reduce the network energy overhead and prolong the network lifetime.
\end{abstract}

\section{Introduction}

The research point is focused on the usage of the least nodes to perform effective coverage of the monitoring area and reduce unnecessary communication between nodes in a network. That is to prolong the lifetime of networks by some strategy under the condition that the coverage rate is satisfied. To some extent, the coverage rate is an important metric to evaluate the network services. Therefore, the coverage rate will be studied in the following four aspects:

First, the network mode for coverage of WSN is proposed.

Secondly, in the view of restraining the energy consumption, the nodes scheduling strategy is introduced to reduce the unnecessary communication path, prolonging the network lifetime.

Finally, the coverage rate curves are given under different parameters by simulations. The proposed algorithm is verified by comparing to other algorithms.

\section{Definitions and Network Model}

The LPOCS algorithm in our paper is based on four assumptions as follows:

(1)The sensor node's sensing range and communication range are both circles;

(2)The communication radius is the twice of sensing radius, and all nodes are connected in the monitored region;

(3)The sensing range of the node is far smaller than coverage region, and the initial energies of nodes are the same and the nodes' clocks are synchronized;

(4)The node position can be obtained through a location algorithm.

\subsection{Basic Definition}

Definition 1.For all nodes which are random deployed in the monitored region, the ratio between their coverage area and the total monitored area is named as Coverage Rate.where $\mathrm{n}$ is the node number, si is the coverage area of each node, and $\mathrm{A}(\Omega)$ is the total monitored area.

$$
P(S, \Omega)=\sum_{i=1}^{n} s_{i} / A(\Omega)
$$


Definition 2. In Two-dimensional monitored region, if a target node is covered or perceived by $\mathrm{K}$ nodes, it is called $\mathrm{K}$ degree Coverage.

Definition 3. Under K degree coverage, the difference between sensor node area and $\mathrm{K}$ degree coverage area is called Effectiveness Coverage Area.si denotes the coverage area of each sensor node, skc represents the coverage area with degree of $\mathrm{k}$.

$$
S_{e c}=\sum_{i=1}^{n}\left(s_{i}-s_{k c}\right)
$$

Definition 4.(Effective Coverage Rate) The ratio between effective coverage area and monitoring area.

$$
P_{e c}=\sum_{i=1}^{n}\left(s_{i}-s_{k c}\right) / A(\Omega)=S_{e c} / A(\Omega)
$$

Theorem 1. Four circles joints with seamless, the maximum effective coverage area is Se=8r2.

\subsection{Network Model}

In this paper, we regard the multiple squares monitoring area as the research object. Supposing that there are two sensor nodes and they satisfy with the relation attribute between adjacent nodes of the Theorem 1. Meanwhile, the square area is divided into three parts which are monitoring area I with significant attention, monitoring area II and unconcerned monitoring area III, as shown in Figure 2. In Figure 2, the side length of area I is $\mathrm{l}$, and that of area II and area III are $\mathrm{l}+\mathrm{r}$ and $\mathrm{l}+2 \mathrm{r}$, respectively. The intersection points between two sensor nodes and area II are A, B, C, D, respectively. The intersection point of the two circles is E.

Definition 5.Any given sensor node si, the ratio of the overlap area of node work perception area and monitoring area accounted for the whole monitoring area, called as the redundant coverage of the node si and si is named redundancy cover node.

Theorem 2. In general, the redundancy cover node si is $n$. The redundancy cover at least satisfies:

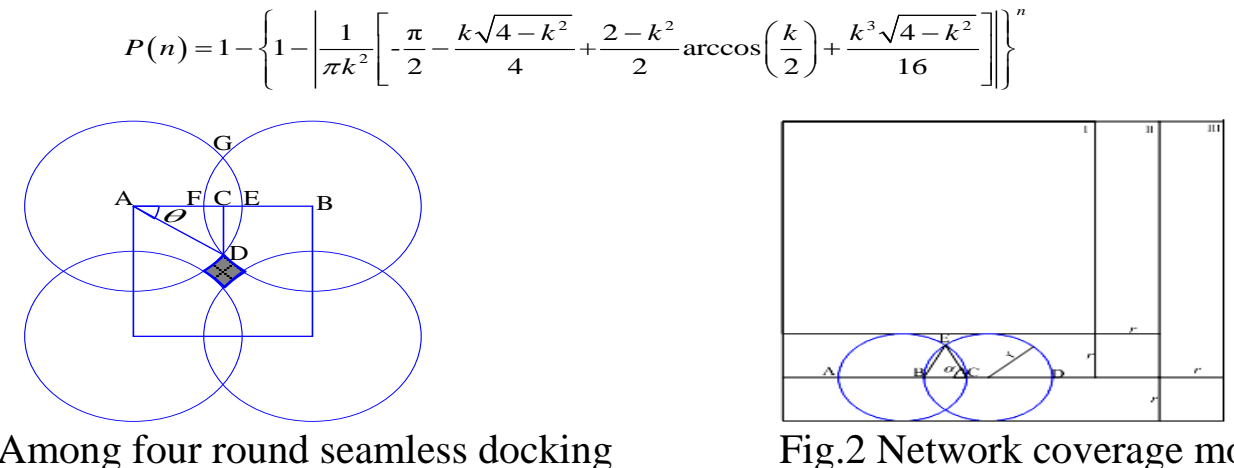

Fig.1 Among four round seamless docking

Fig.2 Network coverage model

Deduction 1.Take the triple square area as the research object, the mathematical expectation of area $I$ is $E(X)=n p 1$, and variance is $D(X)=n p 1(1-p 1)$; Focus on the area $I I, E(Y)=n p 2$, variance $\mathrm{D}(\mathrm{Y})=\mathrm{np} 2(1-\mathrm{p} 2)$. $\mathrm{p} 1$ and $\mathrm{p} 2$ are the coverage of monitoring area I and II, respectively.

\section{Cover quality and algorithm implementation}

\subsection{The analysis of the network coverage quality}

In certain coverage under the premise, in order to further improve the coverage quality, reduce network energy consumption and maximize the network lifetime, to work effectively scheduling of sensor nodes in the monitoring region, so as to save the energy of sensor nodes, and then improve the purpose of network life cycle. For the target nodes in the monitoring region, due to random moving target node will make a number of sensor nodes to a target node coverage, the first to complete the target node coverage expected value depends on the size of the coverage rate and the number of target transfer ratio. 
Deduction 2. In a certain monitoring area, suppose coverage probability of sensor nodes is p. After completing the target node coverage for the first time, its initial expectation of first coverage is $\mathrm{E}(\mathrm{X})=[1-(1-\mathrm{p}) \mathrm{N}] \mathrm{p}-1, \mathrm{~N}$ is the number of target node transfer.

In order to strengthen the effective coverage of the target node is concerned, the energy consumption of sensor nodes in the process, the node energy consumption consists of a sequence of coverage area after the collection of not less than the monitoring area, you can complete the effective coverage of the monitoring area

Theorem 3. On the two dimensional plane, the fitting function of sensor nodes energy consumption is $f(x, y)$. After the energy attenuation the coverage area is $S n$, where $n \in[1,2,3 \ldots N]$. The bounded closed region covers the entire monitoring area, namely:

$$
\iint_{S} f(x, y) d x d y=\lim _{x \rightarrow \infty} \iint_{S_{n}} f(x, y) d x d y
$$

\subsection{The basic idea of the algorithm}

LPOCS algorithm takes the running time of the network as the basic unit, and each round contains two aspects: overlay control information and node status stability information. In the working phase, the working node always remains open, and all redundant nodes are in the closed state to save network energy. In the steady state phase, there are five running states for each node, namely, judgment, competition, waiting, start and sleep. The conversion relationship between them is shown in figure 3. When a monitoring area of the node density is too large, most of the nodes in the region will meet the redundant nodes judging conditions, at the same time, these nodes will enter a dormant state, this state can inhibit the energy consumption of nodes, but there are also some problems, the reason is that the perception of the neighbor node once entered dormancy the state will cause the region to monitor a large number of coverage area, thus reducing the quality of coverage. In order to avoid this situation, the LPOCS algorithm uses a node to wake up its neighbor node and let the neighbors wait in the waiting state when it is asleep. The aim is to reduce the density of possible jobs first, and then to schedule redundant nodes in a candidate job node. Each candidate node elects itself as a pre - working state by probability $\mathrm{P}$, and a candidate without a successful candidate enters the pre sleep state.

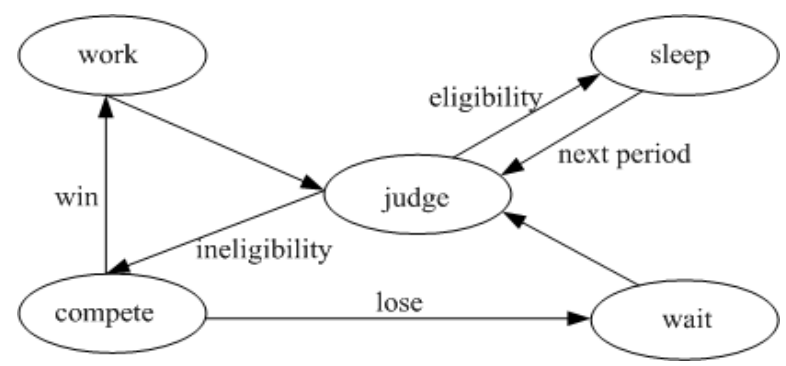

Fig.3 State transition of nodes

\section{System evaluation}

MATLAB7.0 is used as the simulation platform for experiment and analysis. Simulation experiment is that each sensor node is randomly deployed in monitoring area by the radius $r$. The initial energy of each sensor node is the same as $2 \mathrm{~J}$. The sending and receiving data communication model are:

$$
E_{T r}(k, d)=E_{T-\text { elec }} k+E_{\text {amp }}(k, d)=\left\{\begin{array}{l}
E_{T-\text { elec }} k+\varepsilon_{f s} d^{2} k, d<d_{0} \\
E_{T-\text { elec }} k+\varepsilon_{\text {amp }} d^{4} k, d<d_{0}
\end{array}\right.
$$

Among them, ,ET-elec and ER-elec represent the energy consumption of wireless sending and receiving model. $\varepsilon f s$ and $\varepsilon a m p$ respectively denote amplifier energy consumption parameters of free space model and the multipath attenuation model. d0 is proportional factor, and its simulation parameters are as shown in Table 1: 
Table 1 Simulation list

\begin{tabular}{cccc}
\hline parameter & value & parameter & value \\
\hline Monitoring area I & $100 * 100$ & $R$ & $k r$ \\
Monitoring area II & $200 * 200$ & $E_{\mathrm{R} \text {-elec }}$ & $50 \mathrm{~J} / \mathrm{b}$ \\
Monitoring areaIII & $300 * 300$ & $E_{\mathrm{T} \text {-elec }}$ & $50 \mathrm{~J} / \mathrm{b}$ \\
$r$ & $5 \mathrm{~m}$ & $\varepsilon_{\mathrm{fs}}$ & $10(\mathrm{~J} / \mathrm{b}) / \mathrm{m}^{2}$ \\
$\varepsilon_{\text {amp }}$ & $100(\mathrm{~J} / \mathrm{b}) / \mathrm{m}^{2}$ & Initial energy & $2 \mathrm{~J}$ \\
$e_{\min }$ & $0.005 \mathrm{~J}$ & time & $100 \mathrm{~ms}$ \\
\hline
\end{tabular}

Experiment 1: Take $300 * 300 \mathrm{~m} 2$ monitoring area as the research object. Under the same $\mathrm{k}$ value and different coverage probability, the comparative relationship between work node and the number of sensor nodes is as shown in Figure 4:

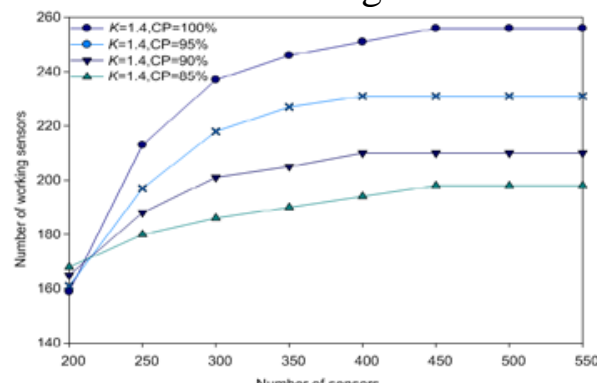

Fig.4(a) $k=1.4$, the number of nodes and working nodes changes graph

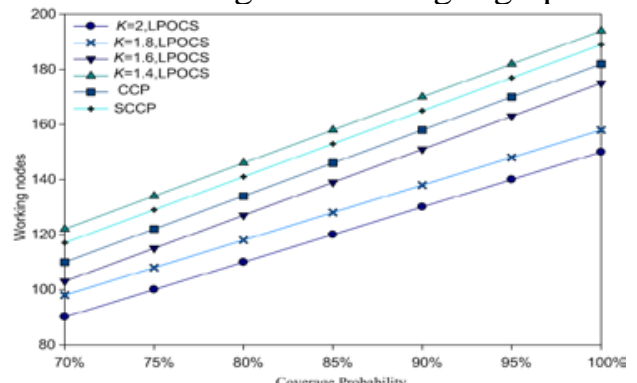

Fig.4(c)200*200m2,Comparison of

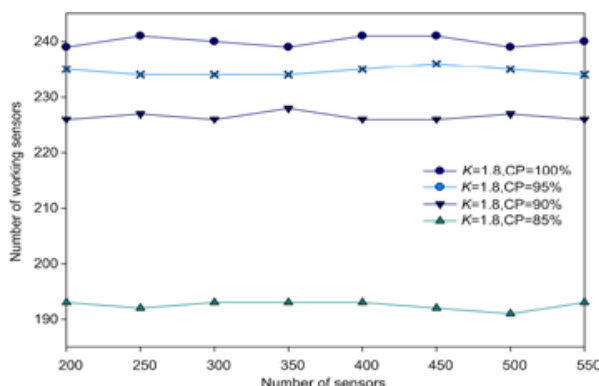

Fig.4(b) $\mathrm{k}=1.8$, the number of nodes and working nodes changes graph

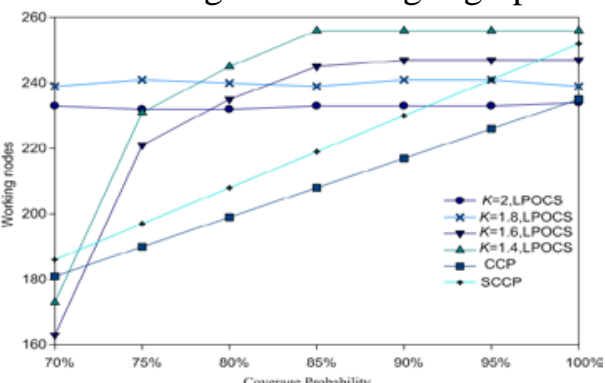

Fig.4(d)300*300m2,Comparison of different algorithms coverage probability

Experiment 2: In order to further verify the network energy equilibrium, The experiment uses two different kinds of monitoring areas, and the process of comparison experiments with different parameters is given under the same conditions. As shown in Figure 5:

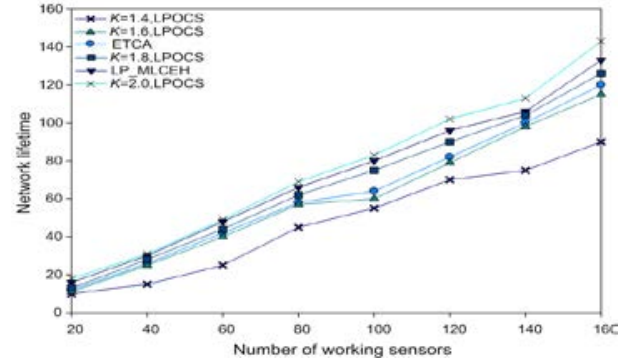

Fig.5(a)200*200m2, Number of sensors and network lifetime changes graph

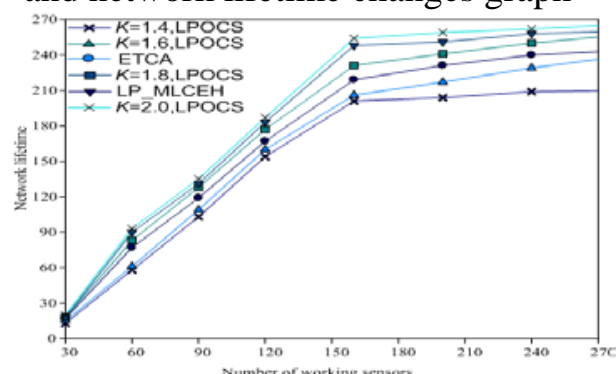

Fig.5(c)300*300m2, Number of sensors and network lifetime changes graph

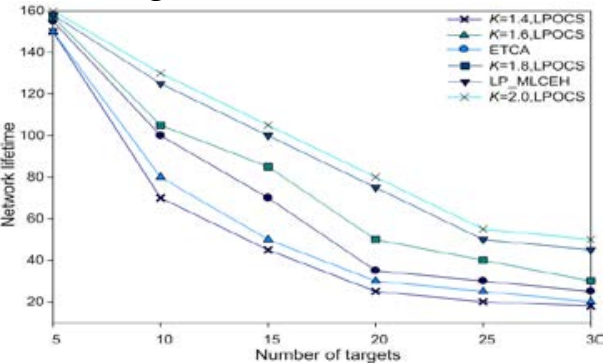

Fig.5(b) Number of targets and network lifetime changes graph

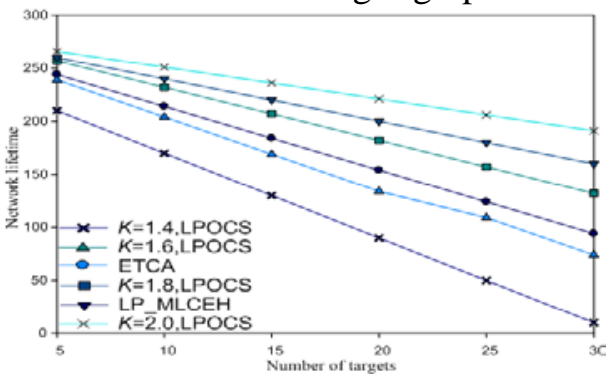

Fig.5(d) Number of targets and network lifetime changes graph 


\section{Conclusion}

In this paper, an optimization covering algorithm based on probabilistic model is proposed. The simulation experiments show the effectiveness and scalability of the LPOCS algorithm. Later work focuses on how to achieve a random two linear regression and how to improve the boundedness of coverage quality.

\section{Acknowledgments}

This work was financially supported by the special science research program fund of Shaanxi Provincial Department of Education, No. 16JK2139

\section{References}

[1]Wang B, Chua KC, Srinivasan V, Wang W. Information coverage in randomly deployed wireless sensor networks. IEEE Transactions on Wireless Communications, 2007, 6(8):2994-3004.

[2]Tseng YC, Chen PY, Chen WT. K-Angle object coverage problem in a wireless sensor network. IEEE Sensors Journal, 2012, 12(12):3408-3416.

[3]Lin JW, Chen YT. Improving the coverage of randomized scheduling in wireless sensor networks. Wireless Communications, IEEE Transactions on, 2008, 7(12): 4807-4812.

[4]Cheng TM, Savkin AV. A distributed self-deployment algorithm for the coverage of mobile wireless sensor networks. IEEE Communications Letters, 2009, 13(11): 877-879.

[5]Tseng YC, Chen PY, Chen WT. k-Angle Object Coverage Problem in a Wireless Sensor Network. Sensors Journal, IEEE, 2012, 12(12): 3408-3416.

[6]Chen A, Kumar S, Lai TH. Local barrier coverage in wireless sensor networks. Mobile Computing, IEEE Transactions on, 2010, 9(4): 491-504.

[7]Li W, Zhang W. Coverage analysis and active scheme of wireless sensor networks. IET wireless sensor systems, 2012, 2(2): 86-91.

[8]Su JS, Guo WZ, Yu CL, Chen GL. Fault-tolerance clustering algorithm with load-balance aware in wireless sensor network. Chinese Journal of Computers, 2014, 37(2): 445-456 (in Chinese with English abstract). 